\title{
A case report of sinoatrial arrest caused by temporal lobe epilepsy in subclinical glioblastoma
}

\author{
Jörg Reifart ${ }^{1 *}$ D, Marlene Tschernatsch ${ }^{2,3}$, Christian W. Hamm ${ }^{1,4}$, Johannes Sperzel ${ }^{1}$ and Andreas Hain ${ }^{1}$
}

\begin{abstract}
Background: Atrial fibrillation with symptomatic bradycardia, higher grade atrioventricular block, and sinus node disease are all common indications for permanent pacemaker implantation. The most frequent causes of sinus node disease treated with pacemaker implantation involve degenerative structural changes of the sinus node; less often, extrinsic causes (such as damage due to myocardial infarction or heightened parasympathetic nervous system activity) lead to pacemaker implantation.
\end{abstract}

Case presentation: A 50-year-old patient with syncope and documented sinoatrial arrest was referred. Neurologic exams (including $\mathrm{CT}$ and $\mathrm{EEG}$ ) revealed no pathologies, so a pacemaker was implanted. Postoperatively, syncope occurred again due to a focal seizure during which sinus rhythm transitioned to atrial pacing by the device. Further neurologic testing revealed focal epilepsy. Six months later, stage IV glioblastoma was diagnosed and the patient was treated surgically.

Conclusion: Intracerebral tumors should be considered in the differential diagnosis for patients with unexplained sinoatrial block, as well as in patients with repeat syncope after pacemaker implantation. Cranial MRI could aid the diagnostic workup of such cases.

Keywords: Cardiology, Sinoatrial arrest, ECG, Glioblastoma, Pacemaker implantation, Bradycardia, Syncope, TLOC

\section{Background}

Alongside higher grade atrioventricular block and atrial fibrillation with symptomatic bradycardia, one of the leading indications for permanent pacemaker implantation is sinus node disease [1]. Treating symptomatic sinus node disease by permanent pacemaker implantation, preferably dual chamber, is strongly recommended (IB) by the European Society of Cardiology (ESC) guidelines [2].

Functional (secondary) sick sinus syndrome is distinguished from a true (primary) sinus node disorder by its extrinsic causes, such as myocardial infarction, electrolyte disturbances, autonomic dysregulation, or adverse

\footnotetext{
* Correspondence: j.reifart@kerckhoff-klink.de

${ }^{1}$ Department of Cardiology, Kerckhoff Heart and Thorax Center, Benekestr.

2-8, 61231 Bad Nauheim, Germany

Full list of author information is available at the end of the article
}

drug reactions. When there is no extrinsic cause, an intrinsic/organic cause is assumed [3].

Bradycardias in association with increased cranial pressure are a well-documented phenomenon [4]. There are some case reports of AV block and asystole occurring with temporal lobe seizures [5].

Here we present a unique case of a sinoatrial block and transient loss of consciousness as the first presenting symptom of subclinical left-sided glioblastoma causing focal temporal lobe epilepsy.

\section{Case presentation}

A 50-year-old otherwise healthy male patient was emergently referred by his primary care physician with recurrent unprovoked syncope which led to multiple hospital visits.

The patient was on beta blocker therapy for supraventricular and ventricular extrasystole, as well as arterial hypertension. There were no other prior medical conditions. 
At the time of admission to our hospital, the patient had experienced four episodes of transient loss of consciousness; the last episode lasted $30 \mathrm{~s}$ and took place in the office of the primary care physician. The first episode took place after mowing the lawn and caused a minor head trauma leading to his admission to the hospital. A head CT after this incident was deemed normal (Fig. 1); the physical and neurologic exams, as well as repeat EEGs revealed no pathologies.

There were no syncope provoking factors. Since there had been a recent death in the family causing the patient increased psychosocial stress, psychogenic pseudosyncope was also considered. After each episode of transient loss of consciousness, the patient was fully alert and oriented. The patient reported no family history of sudden cardiac death or other cardiovascular diseases.

In 24-h Holter monitoring ordered by the primary care physician, a $6 \mathrm{~s}$ sinoatrial arrest had been documented. Later, in our clinic, asystole of $12 \mathrm{~s}$ was documented (Fig. 2). During the episodes, there were no clinical signs of generalized seizure (no involuntary movements, tongue bite, incontinence, postictal confusion).

After ruling out Lyme disease, relevant coronary artery disease, a structural heart disease, and pulmonary embolism, a dual chamber magnetic resonance (MR) conditional pacemaker was implanted.

On the first postoperative day, the patient suffered another transient loss of consciousness. During this episode we observed sinus rhythm transitioning to atrial pacing by the device without a pause on telemetric monitoring. This event prompted a neurological consultation. Again, the clinical neurological exams showed no pathological findings; however during EEG, a hyperventilation provocation test triggered a focal seizure (Fig. 3). Therapy with antiepileptic medication was initiated; at this time the patient's medication list consisted of an angiotensin-convertingenzyme (ACE) inhibitor because of arterial hypertension, and both acetylsalicylic acid and a statin due to coronary artery sclerosis. He was transferred to a neurology clinic.

Subsequent neurological exams in the neurology clinic, including EEG testing for photosensitive epilepsy triggering and a hyperventilation test, as well as duplex sonography of extra- and intracranial arteries showed normal results and the patient was discharged without changes to his medications.

Six months later, the patient presented with focal seizures, aphasia, and recollection difficulties. Stage IV glioblastoma was diagnosed by CT and MRI and treated surgically (Fig. 4).

\section{Discussion and conclusions}

Focal temporal lobe epilepsy has been linked to syncope and bradycardia by several case reports [5-7]. Here, the patient's seizure activity may have been secondary to a very early stage glioma. There were no neurological symptoms upon presentation and initial CT showed no evident signs of the later-diagnosed glioblastoma or of intracranial pressure as the cause of the bradycardia (Fig. 1).

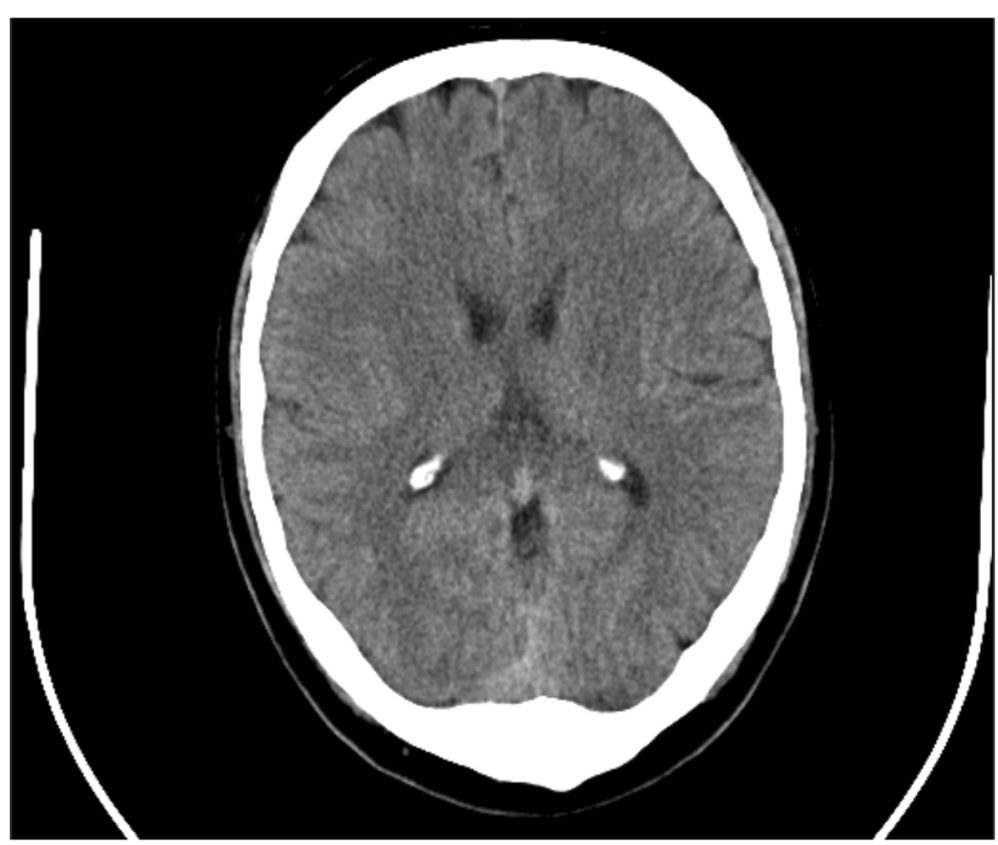

Fig. 1 CT before pacemaker implantation 


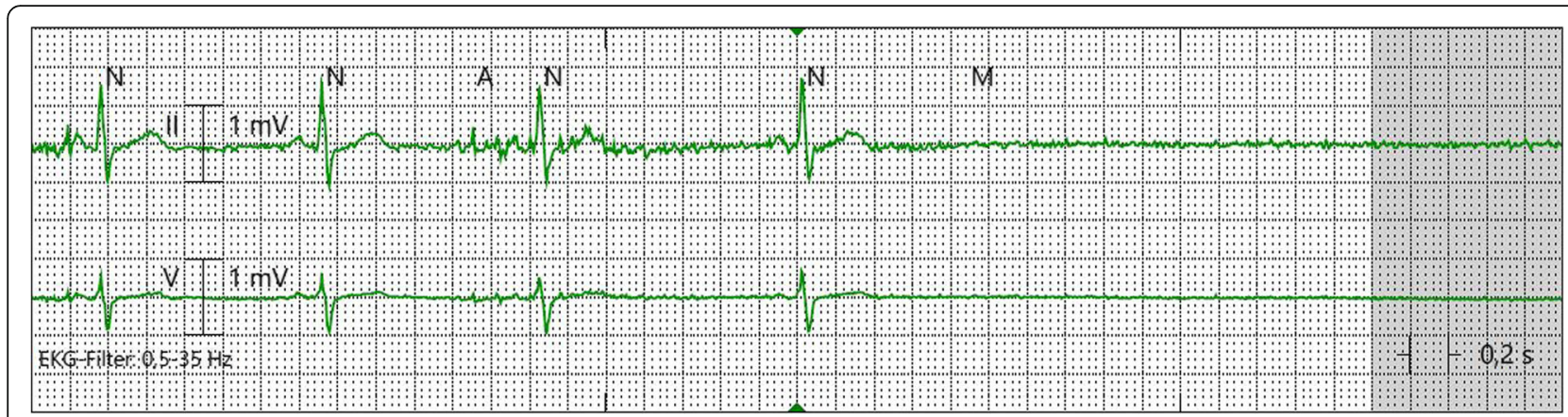

Fig. 2 Sinoatrial block

As standard workup for syncope in patients without neurological signs does not regularly include provocation EEGs or cranial MRI, unusual cases such as this can easily be missed, which can lead to delay in diagnosis when time is critical.

Unfortunately, pacemaker implantation precludes patients from undergoing a MRI study for 6 weeks [8]. In this case, MRI was only performed when symptoms reoccurred while the patient was on antiepileptic medication, which occurred at 6 months post pacemaker implantation; the lack of earlier MRI performed as soon as possible at 6 weeks post pacemaker implantation certainly prolonged the time to glioblastoma diagnosis for this patient.
In contrast to other reports about the influence of epileptic focal seizures on heart rhythm, here the EEG activity preceded the sudden bradycardia [9] (Fig. 2). Proposed mechanisms could include excessive parasympathetic tone or sympathetic inhibition mediated by vagal nerve stimulation during seizure activity leading to the witnessed extrinsic sinus node dysfunction [10].

In hindsight, the focal seizure in this case did most likely predominantly contribute to the transient loss of consciousness with sinus arrest resulting from neuronal suppression.

Sudden unexpected death in patients with epilepsy is not uncommon. Though often the cause of death remains unclear, arrhythmias such as sinus arrest and

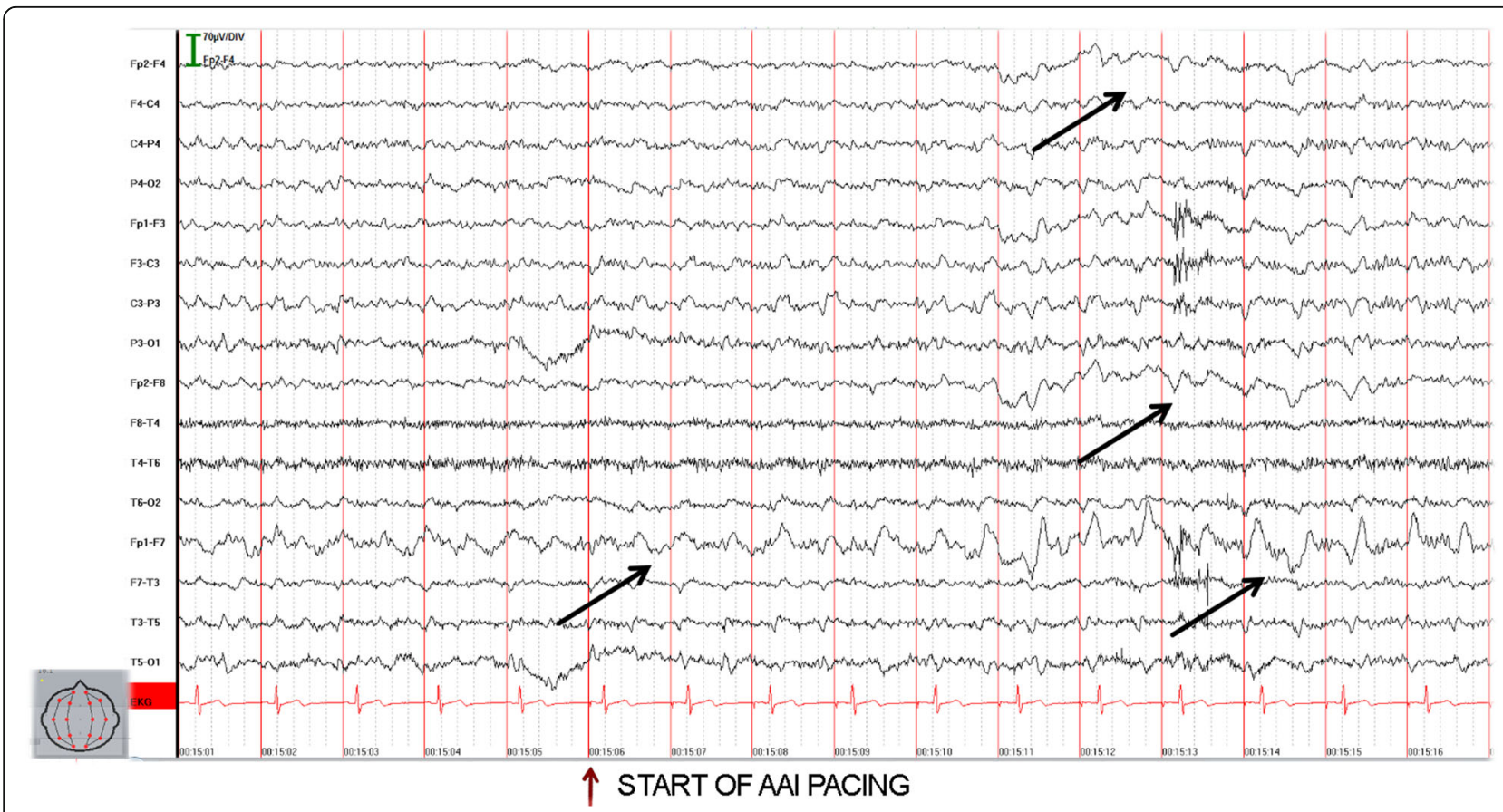

Fig. 3 Seizure activity during which pacing occurs - arrows mark beginning of characteristic EEG activity (rhythmic delta-waves, starting left frontal (Fp1-F7) and later lateralizing to the right side) 


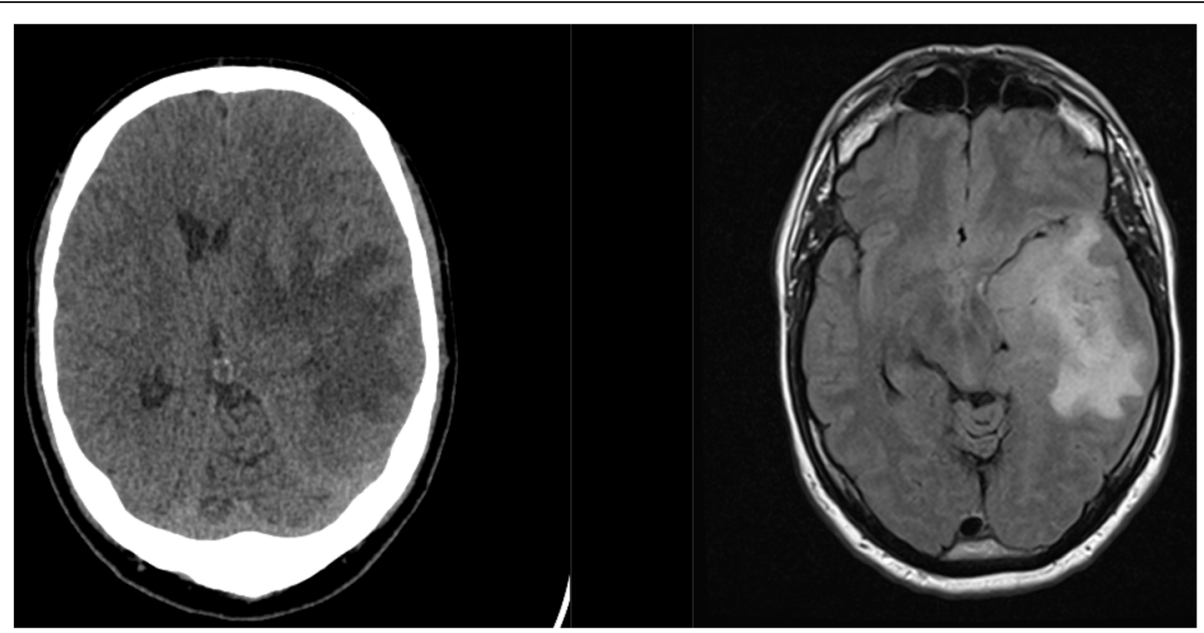

Fig. 46 months after pacemaker implantation; CT (left) and MRI of newly diagnosed glioblastoma

bradycardia are thought to contribute to some cases [11].

The workup of syncope can sometimes be challenging. In patients with sinus arrest of unknown origin, intracerebral tumors should be considered in the differential diagnosis. Repeat loss of consciousness after pacemaker implantation should trigger a more intense neurologic workup, possibly including a cranial MRI if the implanted pacemaker is MR conditional or safe.

\section{Abbreviations}

AV: Atrioventricular; CT: Computer tomography; EEG: Electroencephalogram; ESC: European society of cardiology; MRI: Magnetic resonance imaging

\section{Acknowledgements}

Not applicable

\section{Authors' contributions}

JR collected patient data, obtained consent for publication and wrote the first draft of the article. MT provided the EEG and neurological assessment of this case. $\mathrm{CH}$ and JS consulted on the case and edited the manuscript. $\mathrm{AH}$ analyzed and interpreted the patient and pacemaker specific data and edited the manuscript. All authors read and approved the final manuscript.

\section{Funding}

There is no funding associated with this research.

\section{Availability of data and materials}

Further data and materials may be made available, upon request by the corresponding author, as long as patient anonymity is upheld.

\section{Ethics approval and consent to participate}

An ethics approval for a case report was not deemed necessary.

\section{Consent for publication}

The authors hereby declare that the patient gave written consent for their clinical details and any identifying images to be published in this study.

\section{Competing interests}

The authors declare that they have no competing interests.

\section{Author details}

'Department of Cardiology, Kerckhoff Heart and Thorax Center, Benekestr. 2-8, 61231 Bad Nauheim, Germany. ${ }^{2}$ Department of Neurology, Justus-Liebig University Hospital of Giessen and Marburg, Giessen, Germany.
${ }^{3}$ Gesundheitszentrum Wetterau, Chaumontplatz 1, Bad Nauheim, Germany. ${ }^{4}$ Department of Cardiology, Justus-Liebig University Hospital of Giessen and Marburg, Giessen, Germany.

Received: 1 October 2019 Accepted: 3 January 2020

Published online: 30 January 2020

\section{References}

1. Nowak B, Misselwitz B, Erdogan A, Funck R, Irnich W, Israel CW, et al. Do gender differences exist in pacemaker implantation?--results of an obligatory external quality control program. Europace. 2010;12(2):210-5.

2. Authors/Task Force M, Brignole M, Auricchio A, Baron-Esquivias G, Bordachar P, Boriani G, et al. 2013 ESC Guidelines on cardiac pacing and cardiac resynchronization therapyThe Task Force on cardiac pacing and resynchronization therapy of the European Society of Cardiology (ESC). Developed in collaboration with the European Heart Rhythm Association (EHRA). Eur Heart J. 2013;34(29):2281-329.

3. Bashour TT. Classification of sinus node dysfunction. Am Heart J. 1985 110(6):1251-6.

4. Agrawal A, Timothy J, Cincu R, Agarwal T, Waghmare LB. Bradycardia in neurosurgery. Clin Neurol Neurosurg. 2008;110(4):321-7.

5. van der Sluiis BM, Renier WO, Kappelle AC. Brain tumour as a rare cause of cardiac syncope. J Neuro-Oncol. 2004;67(1-2):241-4.

6. Joske DJ, Davis MJ. Sino-atrial arrest due to temporal lobe epilepsy. Aust NZ J Med. 1991;21(1):62-4.

7. Constantin L, Martins JB, Fincham RW, Dagli RD. Bradycardia and syncope as manifestations of partial epilepsy. J Am Coll Cardiol. 1990;15(4):900-5.

8. Levine GN, Gomes AS, Arai AE, Bluemke DA, Flamm SD, Kanal E, et al. Safety of magnetic resonance imaging in patients with cardiovascular devices. Circulation. 2007;116(24):2878-91.

9. Schernthaner C, Lindinger G, Potzelberger K, Zeiler K, Baumgartner C. Autonomic epilepsy--the influence of epileptic discharges on heart rate and rhythm. Wien Klin Wochenschr. 1999;111(10):392-401.

10. Spear JF, Moore EN. Influence of brief vagal and stellate nerve stimulation on pacemaker activity and conduction within the atrioventricular conduction system of the dog. Circ Res. 1973;32(1):27-41.

11. Goldman AM. Mechanisms of sudden unexplained death in epilepsy. Curr Opin Neurol. 2015;28(2):166-74

\section{Publisher's Note}

Springer Nature remains neutral with regard to jurisdictional claims in published maps and institutional affiliations. 